"Symptoms of Neurologic Disorders" and ending with "Ethical and Legal Guidelines". It has the usual sections on peripheral neuropathies, movement disorders and other classical neurological subdivisions. It is surprisingly comprehensive, and has for example a chapter on neurologic disease during pregnancy. No textbook is perfect and, although this chapter mentions the higher incidence of carpal tunnel syndrome, Bell's palsy and meralgia paresthetica during pregnancy, there is no mention of lumbosacral plexopathy occurring as a result of prolonged labour or forceps delivery.

With its 165 chapters, this is a very comprehensive book. It has an interesting section entitled "Environmental Neurology", which covers issues like alcoholism, drug dependence, and a host of topics including heavy metal intoxication, and falls in the elderly.

In general, for a hard cover textbook, it is very up to date. The chapter on prion diseases for example has a good discussion of the 14-3-3 protein and its usefulness in Creutzfeldt Jakob disease diagnosis in CSF samples, and also a short discussion of new variant Creutzfeldt Jakob disease. The chapter on headache mentions all four of the triptans currently on the market in Canada. Surprisingly, this chapter perpetuates older terminology such as common migraine and classic migraine, and does not use the diagnostic terminology of the International Headache Society.

This is a multi-authored textbook, and the list of contributors runs to eight pages. It is very much an American textbook, with only six contributors from outside the United States. In fact, the great majority of the contributors come from New York.

In summary, this is a well-indexed and useful book. For those who want a hard cover neurology textbook which is succinct, relatively complete, and up to date in a 1000 pages, this book is a good choice.

Werner J. Becker Calgary, Alberta

\section{Normal and Pathologic Development of the Human Brain} ANd Spinal CoRd. 1999. By Maria Dambska, Krystyna E. Wisniewski. Published by John Libbey \& Company Ltd, London, England. 212 pages. C $\$ 118.50$ approx.

This book is by two neuropathologists, and is divided into two parts. The first describes normal development and the second, pathologic development. The first part is somewhat disappointing. Although there is a good description of the embryology of the brain, with excellent black and white drawings of development, the text is somewhat laborious, and not without amphigory. For example, the authors write: "We conclude that neuronal death, which is necessary for the final maturation of connections in the CNS, requires further study." Also "the role of myelin sheaths is apparently complex." Peripheral neurons are referred to as "ganglionic gangliocytes." There are a number of important omissions. For example, olfactory neurons are stated to retain their dividing potential, but not the dentate granule neurons of the hippocampus. This accounts for the sensitivity of the dentate to the mitotic spindler inhibitor colchicine. Also not mentioned are any of the genes of development that are now known. The importance of homeobox genes in rostro-caudal differentiation and sonic hedgehog protein in ventral motoric differentiation of neurons could be given, but they mention only retinoic acid. Nevertheless, the first portion of the book does describe most of the important features of development such as the radial glia and timetable of myelination and gyration. Indeed, Table 3 on myelination is especially useful.

In the second part of the book, the authors do much better, reflecting their experience as neuropathologists. Here, there is authoritative, if brief, coverage of the phakomatoses, CNS malformations, developmental disturbances due to chromosomal aberrations, and late and secondary developmental abnormalities. The justification of this last category, which includes schizencephaly and porencephaly, as separate from the long chapter on malformations of the CNS, is not entirely justified in view of our understanding of these conditions. Indeed, this brief chapter adds only hydranencephaly and cystic encephalopathy to form a loosely coherent, short chapter. The final chapter, entitled "Delay of the CNS maturation" is even shorter, constituting less than two pages. The concept of a disease consisting solely of CNS delay in maturation, is not entirely justified, although the authors refer to numerous conditions including "severe gestosis" or pre-eclampsia of pregnancy. In view of work in the UK, however, I doubt that "chronic hypoxia ... leads particularly often to retardation of myelination of the CNS pathways." Nevertheless, part II contains excellent photographs of cerebella hypoplasia, lissencephaly, heterotopias, holoprosencephaly, agenesis of the corpus callosum, diplomyelia and Chiari malformation. There are fewer photographs of the phakomatoses, but then, this is a short book.

In spite of the above anomalies in English (not the authors'first language), I generally like the book and learned things while reading it. Important questions are addressed even in part I with its omissions, such as invasion of mesenchyme into the ectoderm, which brings not only blood vessels but microglia into the brain. The authors have important considerations in mind, and this is apparent when reading this book. A future edition could be enhanced by correlating with molecular knowledge, tightening the English and style of prose, and adding a few more illustrations of some of the important conditions described.

Roland Auer
Calgary, Alberta

Myasthenia Gravis and Myasthenic Disorders. First Edition. 1999. Edited by Andrew G. Engel. Published by Oxford University Press (Contemporary Neurology Series), New York. 310 pages. C\$157.95 approx.

Engel presents a multi-authored scholarly work detailing current concepts about myasthenia gravis (MG) and myasthenic disorders. The volume is clearly organized in three main parts: approach to diseases of the neuromuscular junction, myasthenia gravis, and myasthenic syndromes and related disorders.

The first part on the approach to diseases of the neuromuscular junction presents much sophisticated experimental work detailing the anatomy and molecular architectures of the neuromuscular junction. An extensive section on the structure and kinetic properties of the acetylcholine receptor follows, with the last chapter outlining the electrodiagnosis of endplate disease. This part of the book proved informative and useful in collating much diffuse material into a compact format which is readily assimilated.

The second part of the book provides information about MG starting with the immunopathogenesis, including a chapter on experimental autoimmune $\mathrm{MG}$, and then progressing to the 
clinically relevant areas of the natural history, diagnosis, and treatment of MG. This section provides a good mix of pathophysiology and information useful for the clinical management of MG patients.

Finally, the third part of the book discusses the more esoteric disorders of the neuromuscular junction, i.e.: the Lambert-Eaton syndrome, neuromyotonia and antiganglioside-associated neuropathies, and the congenital myasthenic syndromes. This section provides a concise review of rarer disorders and is of more specialized interest although general neurologists will be able to use this section for reference purposes.

Each chapter is presented by an authority in the field. Reading this volume will provide the physician with a broader knowledge base which can be used to understand and manage more successfully patients with neuromuscular disorders. Overall the book provides a balanced approach to MG, although a regional bias is apparent in some parts such as the section emphasizing repetitive nerve stimulation studies.

This book is an essential review for any neurologist managing patients with neuromuscular disorders and is highly recommended to anyone wishing to gain a deeper perspective into disorders of the neuromuscular junction.

Vera Bril

Toronto, Ontario

Neurosurgical Classic II. 2000. Edited by Robert H. Wilkins and Gloria K. Wilkins. Published by The American Association of Neurological Surgeons. 592 pages. C\$124.95 approx.

Neurosurgery, like other fields in medicine, is involved in an ever-expanding network of technical information about which it is a difficult enough job to keep informed, let alone be aware of the historical events upon which contemporary knowledge is based. However, neurosurgery is a relatively new specialty so that, until recently, most of us had "lived through" these events and so were reasonably aware of them. But things change and, as the authors state, their book is aimed at "... especially younger neurosurgeons who may not be fully aware of the origins of some aspects of their daily practice". That neurosurgeons are interested in their history is attested to by the success of Class I, presenting 52 papers published before 1940, as well as by the growth of interest in the History of Neurological Surgery at each annual AANS meeting. Furthermore, in order to make advances, it is essential to return to the beginnings before moving on. "This book is dedicated to the neurosurgeons of today who are producing the classics of tomorrow".

Classics II is an attractively bound 592 page compendium of 58 papers published after 1940, divided into 31 subject groups: diagnostic techniques, general surgical and therapeutic techniques (increased intracranial pressure, topical hemostasis, bipolar coagulation, ventriculostomy reservoirs, and cranioplasty), and specific surgical techniques (microneurosurgery, skull base surgery, stereotaxis and radio surgery, and endovascular surgery) following which specific disease entities are discussed (neuro-oncology, occlusive cerebral vascular disease, subarachnoid hemorrhage, head injury, cervical traction and halo immobilization, nerve disorders, hydrocephalus, disc disease, pain and epilepsy). To this reviewer, the most fascinating aspect of Classics II is the compilers' charmingly written introductory commentary for each section reflecting a long, in-depth experience with the literature, and tracing back the roots of each of the 58 selected papers, richly illustrated by quotations from contemporaries, and from an impressive bibliography of their own. The introduction in each case shows the evolution of the thinking involved in the Classics being discussed, and they are as delightful to read as James Burke's "Connections". For example, concerning MRI, the basic discoveries of Rabi, Ramsey, Purcell, Bloch, Bloembergen, Hahn, Ernst, Demadian, and Lauterbur are traced, work that resulted in five Nobel prizes. Along with such information, the parallel development of medicine and magnetism is traced back to Sir William Gilbert's time in the 17th Century.

Canadian readers may be surprised to find that Kenneth McKenzie is credited with developing the first ventriculostomy reservoir to treat tuberculous meningitis with streptomycin, a device this reviewer remembers using. The development of modern electrocoagulation reads like a Tom Swift novel, though I would have included the introduction of radiofrequency lesion making in percutaneous and stereotactic surgery by Sweet and Rosomoff and their associates. Though many of us used the microscope in the neurological laboratory for years, it was apparently 1957 before Ted Kurze did the first microsurgical operative procedure. Again, Canadians will read with interest that Gordon Murray is said to have performed the first successful surgery to restore circulation in an occluded common carotid artery on September 20, 1950. It is surprising how circuitous was the evolution of carotid endarterectomy as we know it today. Endoscopy apparently started in 1910 using urological instruments, and I clearly remember one of my patients spared from a craniotomy in the early 1960s by the use of an arthroscope introduced to our hospital from Japan by Bob Jackson of the orthopaedics staff.

To me, this book is a pleasure to read and an invaluable reference. Naturally, not everyone will agree on the choice of the "Classics". To this reviewer, these should initiate a trend in thought that leads to innovations. Thus, the Classic may not be so original, yet may still be a classic because, unlike some very original contributions, it was published in a situation that inspired those who read it to change history.

Some additional topics that come to mind, not dealt with by the authors, include the discovery of post-traumatic syrinx, the work of Botterell and Lougheed and their associates in 1957 and 1958 with hypothermia and the direct occlusion of aneurysm necks. Under head injury, diffuse axonal injury is reviewed, but the concept of, in my opinion, the more important matter of prophylactic treatment to avoid delayed neuronal injury is not given equal prominence. In the area of functional neurosurgery, I would have included Russell Meyers' open operations on the basal ganglia leading into the modern developments in movement disorder surgery. The field of pain surgery has been revolutionized by the use of chronic stimulation and intrathecal drug installation, which are not treated and the spinal DREZ procedure is a true classic.

This work is an important contribution to any neurosurgeon's library and priority when reading it should be given to the authors' remarkable introductions for each section.

R. Tasker Toronto, Ontario 